\title{
BMJ Open Emergency medical dispatch recognition, clinical intervention and outcome of patients in traumatic cardiac arrest from major trauma: an observational study
}

Craig Prentice, ${ }^{1}$ Jeyasankar Jeyanathan, ${ }^{1,2}$ Richard De Coverly, ${ }^{1}$ Julia Williams, ${ }^{1,3,4}$ Richard Lyon ${ }^{1,5}$

To cite: Prentice C, Jeyanathan J, De Coverly R, et al. Emergency medical dispatch recognition, clinical intervention and outcome of patients in traumatic cardiac arrest from major trauma: an observational study. BMJ Open 2018;8:e022464. doi:10.1136/ bmjopen-2018-022464

\section{- Prepublication history for} this paper is available online. To view these files, please visit the journal online (http://dx.doi. org/10.1136/bmjopen-2018022464).

Received 3 March 2018

Revised 26 June 2018

Accepted 16 August 2018

\section{Check for updates}

(C) Author(s) (or their employer(s)) 2018. Re-use permitted under CC BY-NC. No commercial re-use. See rights and permissions. Published by BMJ.

${ }^{1}$ Kent, Surrey and Sussex Air Ambulance Trust, Redhill, UK ${ }^{2}$ Academic Department of Military Anaesthesia and Critical Care, Defence Medical Services, Birmingham, UK

${ }^{3}$ University of Hertfordshire, Hertfordshire, UK

${ }^{4}$ South East Coast Ambulance

Service, Crawley, UK

${ }^{5}$ University of Surrey, Guildford, UK

Correspondence to Dr Richard Lyon; richardlyon@doctors.org.uk

\section{ABSTRACT}

Objectives The aim of this study is to describe the demographics of reported traumatic cardiac arrest (TCA) victims, prehospital resuscitation and survival to hospital rate.

Setting Helicopter Emergency Medical Service (HEMS) in south-east England, covering a resident population of 4.5 million and a transient population of up to 8 million people.

Participants Patients reported on the initial 999 call to be in suspected traumatic cardiac arrest between 1 July 2016 and 31 December 2016 within the trust's geographical region were identified. The inclusion criteria were all cases of reported TCA on receipt of the initial emergency call. Patients were subsequently excluded if a medical cause of cardiac arrest was suspected.

Outcome measures Patient records were analysed for actual presence of cardiac arrest, prehospital resuscitation procedures undertaken and for survival to hospital rates. Results 112 patients were reported to be in TCA on receipt of the $999 / 112$ call. 51 (46\%) were found not to be in TCA on arrival of emergency medical services. of the 'not in TCA cohort', 34 (67\%) received at least one advanced prehospital medical intervention (defined as emergency anaesthesia, thoracostomy, blood product transfusion or resuscitative thoracotomy). Of the 61 patients in actual TCA, 10 (16\%) achieved return-ofspontaneous circulation. In $45(88 \%)$ patients, the HEMS team escorted the patient to hospital.

Conclusion A significant proportion of patients reported to be in TCA on receipt of the emergency call are not in actual cardiac arrest but are critically unwell requiring advanced prehospital medical intervention. Early activation of an enhanced care team to a reported TCA call allows appropriate advanced resuscitation. Further research is warranted to determine which interventions contribute to improved TCA survival.

\section{INTRODUCTION}

Major trauma is the leading cause of death in young adults worldwide (National Audit Office, 2010). ${ }^{1}$ Historical observational
Strengths and limitations of this study

- This study is unique and one of the first to report on the specific critical issue of bystander recognition of cardiac arrest following trauma.

- The results of this study have direct implications for the prehospital tasking of trauma teams.

- Implementing the conclusion of this study, that early, accurate tasking of trauma teams to reported traumatic cardiac arrest is warranted, could save many lives.

- The study is retrospective and further prospective research is warranted in this area.

- The external validity of this study remains to be fully assessed and further research is warranted in other emergency medical services.

studies report survival rates from traumatic cardiac arrest (TCA) being between $0 \%$ and $2.3 \% .^{2}{ }^{3}$ The mortality rate from TCA is high. ${ }^{45}$ Sherren et al (2013) suggest that resuscitation from TCA, despite common misconception, is not universally futile with recent publications suggesting more favourable survival rates. ${ }^{56}$

In TCA, advanced prehospital medical care can bring clinical interventions such as advanced airway management, thoracostomy, resuscitative thoracotomy and blood product transfusion. ${ }^{7-9}$ These procedures are not routinely available to standard land ambulance crews and all can have a direct effect on patient outcome from TCA. ${ }^{8}$ In order to deliver rapid advanced medical care in the prehospital phase, enhanced medical care teams, such as Helicopter Emergency Medical Services (HEMS) need to be dispatched early to possible TCA cases. ${ }^{10} 11$

Emergency medical dispatch to possible TCA cases is challenging. ${ }^{1213}$ Patients who are 
critically injured following trauma are often mistaken by members of the public as being in cardiac arrest when the initial emergency call is made. However, these patients can frequently be critically unwell and have a window of opportunity for lifesaving interventions. If these interventions are not performed in a timely manner, the patient may rapidly progress to traumatic cardiac arrest and mortality subsequently increased. ${ }^{5}$ In order to prevent deterioration or achieve a return-of-spontaneous circulation (ROSC) following TCA, early dispatch and rapid response of HEMS teams are required. ${ }^{13}$

The host National Health Service (NHS) Ambulance Service uses the NHS Pathways System (NHSP) for 999 call assessment and prioritisation. ${ }^{14}$ Immediately life-threatening calls received by the Ambulance Service were split into Red 1 and Red 2 subcategories. The Red 1 category includes patients with the most time critical elements reported in the 999 call. In NHSP, there are two disposition codes that generate a Red 1 coded emergency incident. Any patient who is reported as not breathing, choking, fitting or who is unconscious with noisy or abnormal breathing will be assigned a Red 1 code and 'dispatch for potential cardiac arrest' disposition. This call subset includes patients with trauma and these incidents are passed to the HEMS service as a potential traumatic cardiac arrest situation. It is therefore to be expected that a proportion of the reported TCA dispatches are actually found not to be in TCA on the arrival of the HEMS service.

The aim of this study is to describe the cohort of patients reported to be in TCA on receipt of the initial emergency call to South East Coast Ambulance Service NHS Foundation Trust. It was hypothesised that many patients who were reported during the emergency call to be in TCA may not be in actual cardiac arrest but could be in a critical condition requiring rapid advanced medical intervention. This study describes this patient demographic, interventions performed and survival to hospital.

\section{METHODS}

A retrospective database analysis of patient demographics, clinical care and outcome was performed. Patients reported to be in TCA between 1 July 2016 and 31 December 2016 within the Trust's geographical region were identified and studied from the local ambulance trust's electronic patient database. The inclusion criteria were all cases of reported TCA on receipt of the initial emergency call. Patients were subsequently excluded if a medical cause of cardiac arrest was suspected. The clinical records of all cases were examined and patient demographics, physiological state, clinical interventions and ROSC analysed.

\section{Study setting}

The Kent, Surrey and Sussex Air Ambulance Trust (KSSAAT) operates in the south-east of England providing a Helicopter Emergency Medical Service
(HEMS) as a paramedic-physician team, responding 24/7 from two separates bases. This serves a static population of approximately 4.3 million and a transient population of up to a total of 10 million. This area consists of both urban and rural areas with Major Trauma Centres in London, Brighton and Southampton. Patient transport to hospital can be by air or road, depending on geography, weather, time of day and hospital helipad availability. On average, patients are $44 \mathrm{~km}$ in linear distance from incident scene to receiving hospital and have an average primary transfer time of $30 \mathrm{~min}$ to hospital by air or road. As well as the speed of reaching these patients, additional advanced clinical interventions are brought to the scene by the HEMS team. These include, but are not limited to, rapid sequence induction (RSI) of anaesthesia, simple and tube thoracostomy insertion, resuscitative thoracotomy, emergency hysterotomy, blood product transfusion, emergency reversal of anticoagulation, and maxillofacial packing. One immediate dispatch criterion is major trauma and/or TCA as evidenced during the initial emergency call description. Interrogation of the call is performed by call handlers who are traditionally not medically trained personnel. Accurately evaluating which patients are in actual or impending TCA from the initial emergency call is crucial if the correct emergency medical response is to be activated.

\section{Outcome measures}

The outcome measures included the proportion of patients initially reported as being in TCA who were subsequently found not to be in cardiac arrest; the rate of HEMS clinical interventions in this patient cohort and survival to hospital.

HEMS advanced interventions were defined as clinical interventions that a land-based ambulance crew would not be able to perform. These included RSI, surgical chest decompression (thoracostomy), transfusion of blood products and resuscitative thoracotomy. Routine interventions were defined as those that could be performed by a UK registered paramedic. Whether the patient was transported to hospital, the transport means (land ambulance vs helicopter) and whether the HEMS crew was required to accompany the patient were also noted.

\section{Patient and public involvement}

This study question was raised, as the outcome from traumatic cardiac arrest remains very poor, with few patients surviving to reach discharge from hospital. Several patients visiting KSSAAT expressed support for the Trust to undertake research in this domain. As well as publication, we plan to disseminate our results via the South East Coast Ambulance Service Research and Development Committee, which includes patient representatives.

\section{Data}

Data was extracted from KSSAAT's bespoke database (HEMSbase, MedicOne Systems Ltd, UK) and analysed on Microsoft Excel (Microsoft Corporation, USA). This 
Table 1 Demographics of the 51 patients with spontaneous circulation who had initially been reported to be in traumatic cardiac arrest

\section{(A) Gender demographics}

\begin{tabular}{ll}
\hline Male & Female \\
\hline 31 & 20 \\
\hline (B) Age ranges & Number \\
\hline Age range & 1 \\
\hline $0-4$ & 1 \\
$5-9$ & 2 \\
$10-19$ & 12 \\
\hline $20-29$ & 5 \\
$30-39$ & 7 \\
$40-49$ & 7 \\
$50-59$ & 5 \\
$60-69$ & 7 \\
$70-79$ & 4 \\
\hline $80-89$ & \\
\hline $90-99$ & \\
\hline
\end{tabular}

(C) Pulse status on primary assessment as a marker of injury severity

\begin{tabular}{ll}
\hline $\begin{array}{l}\text { Pulse status on primary number } \\
\text { assessment }\end{array}$ & Number \\
\hline Radial pulse & 43 \\
Femoral pulse & 4 \\
Carotid pulse & 4 \\
\hline
\end{tabular}

study met National Institute for Healthcare Research criteria for a service evaluation and was registered with the University of Surrey, as such formal ethical approval for a service evaluation was not required and waived.

\section{RESULTS}

The database review found 160 patients that were reported as being in 'cardiac arrest' on receipt of the 999/112 emergency call. Of these, 48 (30\%) were subsequently found to be primary medical arrests and were therefore excluded. This left 112 patients being reported from the 999 call as being in TCA.

On arrival of land ambulance crews and/or the HEMS team, $61(54 \%)$ were found to be in actual cardiac arrest. Of the 112 patients reported to be in TCA, 51 (46\%) had spontaneous circulation on arrival of the first emergency medical services. The demographic details of these 51 casualties are presented in table 1A-C. Patient inclusion is shown in figure 1.

\section{Prehospital clinical interventions}

Following arrival of emergency medical services, the prehospital clinical interventions administered to the patient were reviewed. 'Routine' and 'HEMS' interventions are shown in table 2.

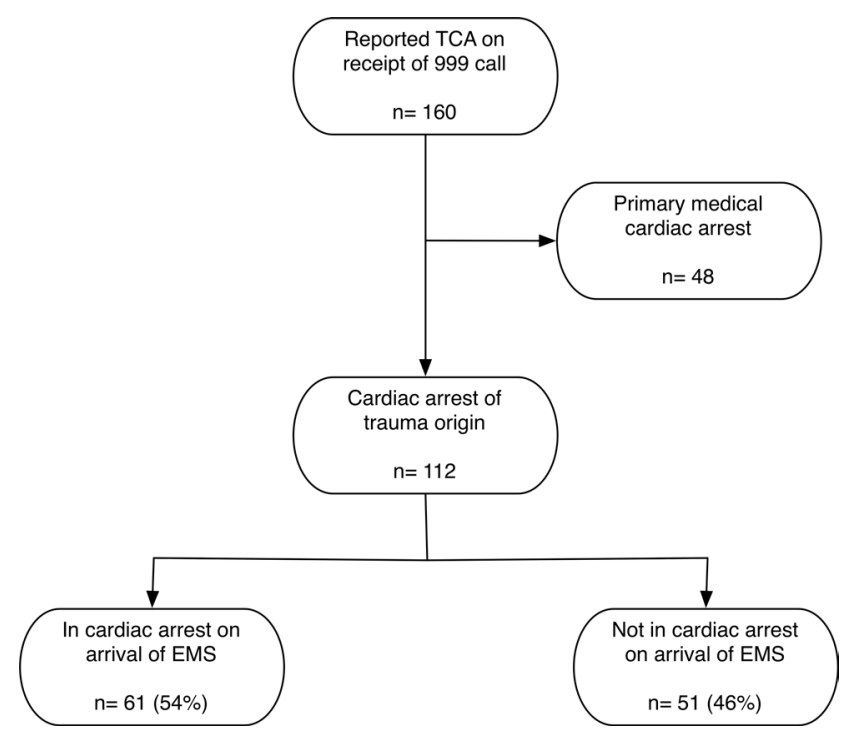

Figure 1 Patient inclusion. EMS, emergency medical services; TCA, traumatic cardiac arrest.

Of the patients not found to be in TCA $(n=51)$, a significant proportion $(n=42,82 \%)$ required advanced clinical intervention from the attending HEMS team. The number of included patients who received each of the HEMS advanced interventions is shown in table 3 .

The total number of HEMS interventions each individual patient received from the HEMS team is shown in table 4.

\section{Patients with cardiac output on arrival of HEMS}

The majority of patients found to have spontaneous circulation required a HEMS escort to hospital. In 24 (47\%) cases, the patient was escorted in a land ambulance; in $21(41 \%)$ cases transported via helicopter and five $(10 \%)$ transported unescorted by the HEMS team with a land ambulance crew. In one case, a patient who initially had spontaneous circulation suffered a cardiac arrest in the presence of the HEMS crew and was subsequently pronounced life extinct at scene.

\section{Patient outcome}

Of the 51 patients initially reported to be in TCA who had spontaneous cardiac output on first EMS arrival, 50 $(98 \%)$ survived to hospital admission. Out of the patients

Table 2 Prehospital clinical interventions

Helicopter Emergency
Medical Services
interventions

Routine interventions interventions

Patient packaging and Rapid sequence induction of immobilisation anaesthesia

Limb splintage Blood product transfusion

Basic airway management Thoracostomy

Intravenous access Resuscitative thoracotomy

Intraosseous access

External haemorrhage control

Pelvic splintage 
Table 3 Helicopter Emergency Medical Services clinical interventions performed in patients with spontaneous circulation who had initially been reported to be in traumatic cardiac arrest

\begin{tabular}{ll}
\hline Intervention & Patients (n) \\
\hline Rapid sequence induction & 26 \\
Blood product transfusion & 16 \\
Thoracostomy & 14 \\
Resuscitative thoracotomy & 0 \\
\hline
\end{tabular}

found to be in actual TCA ( $n=61)$, prehospital ROSC was achieved in $10(16 \%)$, with all these patients surviving to reach hospital.

\section{DISCUSSION}

This study found that $46 \%$ of patients who were reported by the bystander on scene to be in TCA following a serious accident, actually had spontaneous circulation on arrival of emergency medicine services. This group of patients had a very high likelihood of needing advanced prehospital clinical intervention, with $82 \%$ of patients who were not in actual TCA requiring one or more clinical interventions from the attending HEMS team.

Previous studies have suggested that resuscitation from TCA has been historically perceived as futile. ${ }^{315} \mathrm{~A}$ risk exists, therefore, that advanced emergency medical services, such as HEMS, will not be dispatched immediately following receipt of a suspected TCA call. As this study has demonstrated, this group of patients are at high risk of being critically injured; with a high proportion needing advanced prehospital clinical care. Any delay in administering advanced care, such as emergency anaesthesia, surgical chest decompression or volume resuscitation, is likely to have a detrimental effect on patient outcome. ${ }^{16}$ When the patient was found to be in actual TCA, clinical intervention from the HEMS team resulted in a ROSC rate of $16 \%$. It would therefore appear prudent to task advanced emergency medical services immediately.

The ability of a layperson to inaccurately diagnose cardiac arrest is well recognised. ${ }^{11} 17$ Accurately diagnosing cardiac arrest following trauma is known to be particularly problematic. Following a traumatic incident,

Table 4 Total number of Helicopter Emergency Medical Service (HEMS) interventions performed in patients with spontaneous circulation who had initially been reported to be in traumatic cardiac arrest

\begin{tabular}{|ll}
\hline $\begin{array}{l}\text { Number of HEMS interventions } \\
\text { performed }\end{array}$ & Patients (n) \\
\hline 1 & 19 \\
\hline 2 & 16 \\
3 & 7 \\
4 & 0 \\
\hline
\end{tabular}

such as a serious road traffic collision, there is often a high degree of emotion and anxiety. The caller themselves may have been involved in the accident, may be a friend or relative of the critical patient, or may themselves be injured. ${ }^{13}$ The ability of a bystander at the scene of a suspected traumatic cardiac arrest to make an accurate assessment may therefore be hampered. ${ }^{1}$ While the current early dispatch of HEMS is based on essentially a symptom-based system, other potential factors could be incorporated to help this, for example, the mechanism of incident or injury, the nature and severity, for example, high-speed road traffic accident, rollover and entrapped casualties.

Previous studies have shown the benefit of HEMS intervention for TCA. ${ }^{6}$ Current guidelines advocate a standard, systematic approach to resuscitation from TCA. ${ }^{8}$ Some elements of the resuscitation algorithm (intubation, surgical chest decompression and blood product administration) can only be performed by advanced care providers, and in some systems, these interventions are limited to physicians. The outcome benefit of specific prehospital clinical interventions in major trauma remains the subject of ongoing research. Early prehospital anaesthesia has been shown to improve outcome in blunt neurotrauma. ${ }^{18}$ In order to maximise the chance of patient survival, these interventions need to be performed as early as possible, if ROSC is to be achieved. ${ }^{1}$ This requires prehospital intervention, with early, accurate identification of the critical medical need at the point of receiving the emergency phone call.

\section{LIMITATIONS}

This study has several limitations. The time period ( 1 July 2016-31 December 2016) for case review was relatively limited; however, the total number of patients included was felt to be reasonable. It was not possible to analyse exact ambulance dispatch system entries to look for specific patterns of recognition of TCA. In particular, it was not possible to analyse the exact voice recordings of the emergency call. Voice call analysis has previously been shown to be valuable in examining telephone recognition of cardiac arrest and would be a valuable area for future research to establish whether specific triggers can inform appropriate resource dispatch to potential cardiac arrest cases. ${ }^{19}$ Other future research could involve the live transmission of mobile phone visual footage from scene to assist in trauma dispatch and patient assessment via telephone.

\section{CONCLUSION}

A significant proportion of patients with major trauma reported to be in TCA on receipt of the initial emergency call are not in actual cardiac arrest but are critically unwell requiring advanced prehospital medical intervention. Early activation of an enhanced care team to a reported TCA call allows appropriate advanced 
resuscitation to occur in the prehospital phase and maximises the patient's chances of meaningful survival. Further research is warranted to determine which prehospital clinical interventions contribute to improved TCA survival.

Acknowledgements We wish to thank all staff at KSSAAT for contributing to ongoing research and development.

Contributors $\mathrm{CP}$ and $\mathrm{JJ}$ were involved in data collation and analysis. Data analysis was reviewed by JW, JJ and RL. All authors contributed to the data review, preparation of the manuscript and approval of the manuscript prior to submission.

Funding The authors have not declared a specific grant for this research from any funding agency in the public, commercial or not-for-profit sectors.

Competing interests CP, JJ, JW, RDC and RL are all employees of Kent, Surrey and Sussex Air Ambulance Trust.

Patient consent Not required.

Ethics approval This study met UK National Institute for Health Research criteria for a service evaluation. All the data utilised for this study was routinely collected as part of standard pre-hospital and hospital patient data collection. Formal ethical approval was therefore waived as criteria for service evaluation were met. The study was registered with the University of Surrey as a service evaluation.

Provenance and peer review Not commissioned; externally peer reviewed.

Data sharing statement All data generated or analysed during this study are included in this published article.

Open access This is an open access article distributed in accordance with the Creative Commons Attribution Non Commercial (CC BY-NC 4.0) license, which permits others to distribute, remix, adapt, build upon this work non-commercially, and license their derivative works on different terms, provided the original work is properly cited, appropriate credit is given, any changes made indicated, and the use is non-commercial. See: http://creativecommons.org/licenses/by-nc/4.0/.

\section{REFERENCES}

1. Evans CC, Petersen A, Meier EN, et al. Prehospital traumatic cardiac arrest: management and outcomes from the resuscitation outcomes consortium epistry-trauma and PROPHET registries. J Trauma Acute Care Surg 2016;81:285-93.

2. Beck B, Bray JE, Cameron P, et al. Predicting outcomes in traumatic out-of-hospital cardiac arrest: the relevance of Utstein factors. Emerg Med J 2017;34:786-92.

3. Rosemurgy AS, Norris PA, Olson SM, et al. Prehospital traumatic cardiac arrest: the cost of futility. J Trauma 1993;35:468-73.
4. Harris T, Thomas GO, Brohi K. Early fluid resuscitation in severe trauma. BMJ 2012;345:e5752.

5. Sherren PB, Reid C, Habig K, et al. Algorithm for the resuscitation of traumatic cardiac arrest patients in a physician-staffed helicopter emergency medical service. Crit Care 2013;17:308.

6. Lockey D, Crewdson K, Davies G. Traumatic cardiac arrest: who are the survivors? Ann Emerg Med 2006;48:240-4.

7. Davies GE, Lockey DJ. Thirteen survivors of prehospital thoracotomy for penetrating trauma: a prehospital physician-performed resuscitation procedure that can yield good results. J Trauma 2011;70:E75-E78.

8. Lockey DJ, Lyon RM, Davies GE. Development of a simple algorithm to guide the effective management of traumatic cardiac arrest. Resuscitation 2013;84:738-42.

9. Lyon RM, de Sausmarez E, McWhirter E, et al. Pre-hospital transfusion of packed red blood cells in 147 patients from a UK helicopter emergency medical service. Scand J Trauma Resusc Emerg Med 2017;25:12.

10. Marung H, Jakisch B, Gräsner J-T, et al. Severe underuse of dispatch life support in traumatic cardiac arrest. Resuscitation 2017;118:e33-e34

11. Viereck S, Møller TP, Rothman JP, et al. Recognition of out-ofhospital cardiac arrest during emergency calls - a systematic review of observational studies. Scand J Trauma Resusc Emerg Med 2017;25:9.

12. Alfsen D, Møller TP, Egerod I, et al. Barriers to recognition of out-ofhospital cardiac arrest during emergency medical calls: a qualitative inductive thematic analysis. Scand J Trauma Resusc Emerg Med 2015;23:70

13. Eaton G, Brown S, Raitt J. HEMS dispatch: A systematic review. Trauma 2018;20:3-10.

14. NHS Digital. NHS Pathways [Internet]. https://digital.nhs.uk/services/ nhs-pathways (cited 19 Jun 2018).

15. Willis CD, Cameron PA, Bernard SA, et al. Cardiopulmonary resuscitation after traumatic cardiac arrest is not always futile. Injury 2006;37:448-54.

16. Pham H, Puckett $Y$, Dissanaike S. Faster on-scene times associated with decreased mortality in Helicopter Emergency Medical Services (HEMS) transported trauma patients. Trauma Surg Acute Care Open [Internet]. 20171 https://www.ncbi.nlm.nih.gov/pmc/articles/ PMC5887760/

17. Clegg A, Young J, Iliffe S, et al. Frailty in elderly people. The Lancet 2013;381:752-62.

18. Bernard SA, Nguyen V, Cameron $P$, et al. Prehospital rapid sequence intubation improves functional outcome for patients with severe traumatic brain injury: a randomized controlled trial. Ann Surg 2010;252:959-65.

19. Clegg GR, Lyon RM, James S, et al. Dispatch-assisted CPR: where are the hold-ups during calls to emergency dispatchers? A preliminary analysis of caller-dispatcher interactions during out-ofhospital cardiac arrest using a novel call transcription technique. Resuscitation 2014;85:49-52. 